\title{
Detection of Epidermal Growth Factor Receptor Dimers on Wet and Intact Eukaryotic Cells in an Environmental Scanning Electron Microscope
}

\author{
D. Peckys, ${ }^{1}$ U. Werner ${ }^{1}$ and N. de Jonge ${ }^{1,2}$ \\ ${ }^{1}$ INM Leibniz Institute for New Materials, Saarbrücken, Germany \\ ${ }^{2}$ Department of Molecular Physiology and Biophysics, Vanderbilt University School of \\ Medicine, Nashville, TN, USA
}

Studying the stoichiometry, i.e., the composition of proteins in protein complexes is presently limited by the available microscopy methods. Cellular macromolecules are mostly imaged with transmission electron microscopy (TEM) yielding nanometer resolution on conventional thin sections, cryo sections, membrane sheets, or freeze-fracture samples, or via serial sectioning. In particular sectioning remains challenging regarding artifacts. Intact cells in liquid are traditionally imaged with light microscopy. But even recently developed super resolution fluorescence techniques lack about an order of magnitude in their spatial resolution as required for resolving the subunits of macromolecular complexes. We have used environmental scanning electron microscopy (ESEM) of whole fixed cells in hydrated state in combination with specific labeling with gold nanoparticles for the study of the epidermal growth factor receptor (EGFR). The EGFR is a transmembrane protein with high relevance in cancer research [1]. The spatial arrangement of EGFRs directly correlates with receptor activation and controls its interaction with other protein partners. Of particular interest is the formation of the EGFR dimer.

Two different cell types (COS7 and A549) were grown on microchips with electron transparent silicon nitride ( $\mathrm{SiN}$ ) windows, and subsequently incubated for 5 minutes with a conjugate of epidermal growth factor (EGF) and a gold nanoparticle (AuNP) of $12 \mathrm{~nm}$ diameter. The cells were then chemically fixed, cooled to $3{ }^{\circ} \mathrm{C}$, transferred into pure water, and placed in the environmental scanning electron microscope (ESEM) (FEI Quanta 400 FEG). The ESEM was equipped with a scanning transmission electron microscopy (STEM) detector underneath the sample, and a gaseous secondary electron (GSE) detector mounted above the sample; the detectors were used synchronously. The cells were imaged in hydrated state in a wet environment of saturated water vapor at a pressure around $740 \mathrm{~Pa}$. High contrast was obtained on the gold labels using the STEM detector [2], while the presence of a thin water layer over the cell was monitored with the GSE detector (Fig. 1A).

Fig. 1B represents an ESEM image recorded at a magnification of $50,000 \times$ sufficient to resolve the individual labels. The spatial resolution measured from line-scans in the images at the locations of AuNPs amounted to $3 \mathrm{~nm}$. Pairs of labels were visible, and were attributed to EGFR dimers, larger clusters were also present. The electron dose for this image was $150 \mathrm{e}^{-}$ $/ \mathrm{nm}^{2}$, only a factor of two larger than that used for cryo TEM studies of cells. It was verified in control experiments that electron beam irradiation did not change the measured distances between the labels in AuNP clusters. The positions of AuNPs in a series of images from different A549 and COS7 cells were automatically detected, and used for further analysis. We have analyzed the density distribution for groups of clusters, i.e., single AuNPs, dimers, and higher order clusters. We have also calculated the pair correlation function, from which the most frequently occurring distances between the centers of the AuNP labels in the different categories of clusters were determined. In case of the EGFR dimer, the measured most probable distance of $19 \mathrm{~nm}$ corresponded to a molecular model. 


\section{References:}

[1] A. Arkhipov et al, Cell 152 (2013), p. 557.

[2] N. de Jonge et al de Jonge, Proc. Natl. Acad. Sci. 106 (2009), p. 2159.

[3] We thank J.P. Baudoin, M. Eder, M. Koch for help with the experiments, A. Kraegeloh for support of the experiments, and Protochips Inc, NC, USA for providing the microchips with silicon nitride support windows. We thank E. Arzt for his support through INM. Research in part supported by NIH grant 2R44-EB008589 (to J. Damiano).

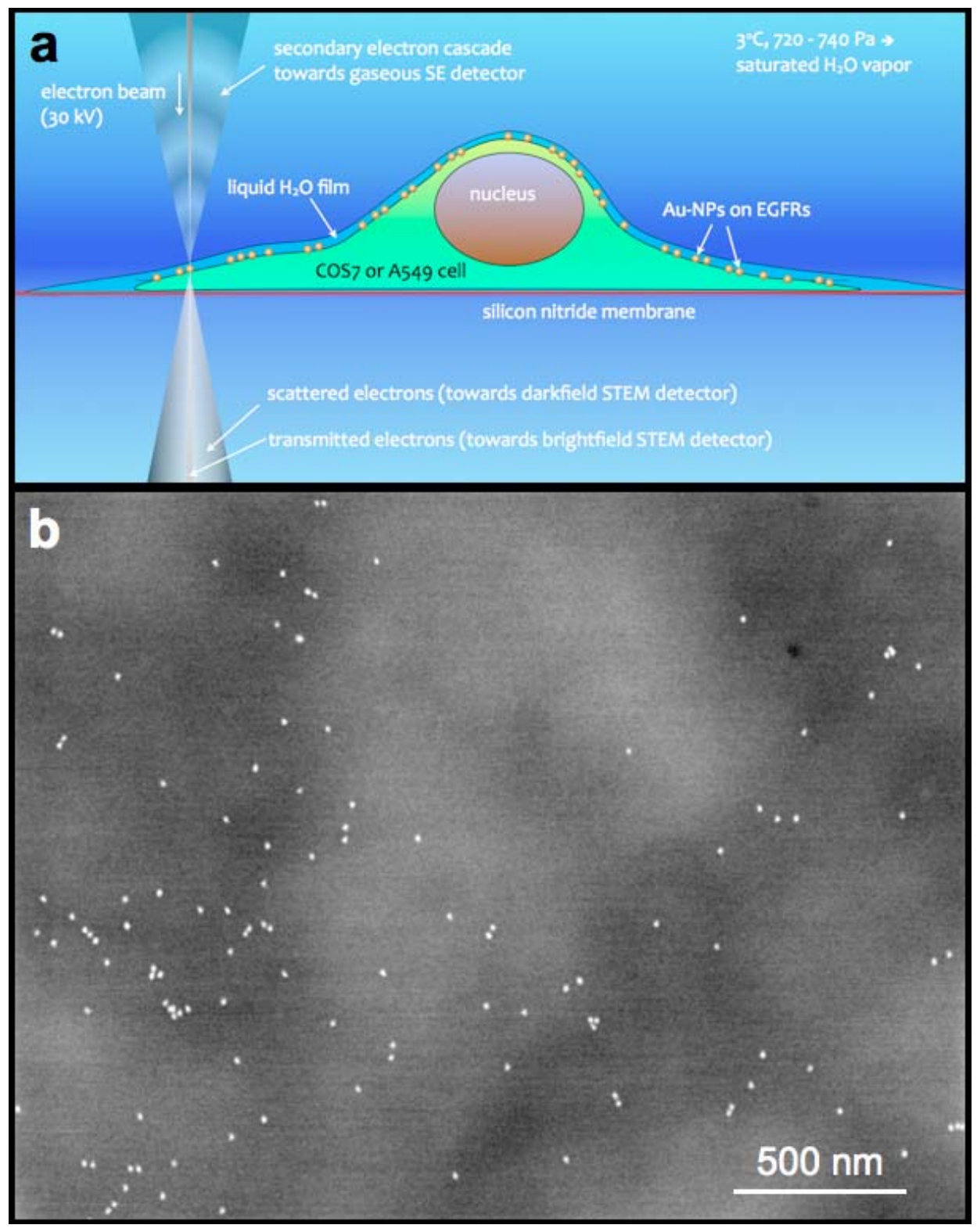

a) Schematic representation of environmental scanning electron microscopy (ESEM) of a a whole eukaryotic cell in wet state. EGFRs on the cell membrane were labeled with Au-NPs. After fixation the cell is maintained in a saturated water vapor atmosphere under a thin layer of deionized water ( $\sim 500 \mathrm{~nm}$ thick). Imaging is done by scanning a focused electron beam over the cell, mostly at its thinner regions. A gaseous secondary electron detector (GSED) is located above the sample, and a scanning transmission electron microscopy (STEM) detector is located beneath the sample. b) STEM image (dark field mode) showing AuNP labeled EGFRs on a A449 cell, revealing smaller clusters and paired labels. 